\title{
Aggressive Embryonal Orbital Rhabdomyosarcoma in an Adolescent
}

\author{
Jacqueline Yih Ling Ting ${ }^{1}$, Norlaila Talib², Mushawiahti Mustapha ${ }^{3}$ \\ ${ }^{1,3}$ Department of Ophthalmology, Universiti Kebangsaan Malaysia Medical Centre (UKMMC), Jalan Yaacob Latiff \\ Cheras, Kuala Lumpur, Malaysia. ${ }^{2}$ Department of Ophthalmology, Hospital Serdang, Selangor, Malaysia.
}

\section{INTRODUCTION}

Rhabdomyosarcoma is a highly malignant neoplasm originating mainly from undifferentiated mesenchymal tissue. It is one of the commonest soft tissue neoplasms in the head and neck region, with an incidence of 4.3 cases per million. ${ }^{1}$ Approximately $10 \%$ of the cases reported occur in the orbit. It is considered a disease of young children with strong male prevalence. ${ }^{2}$ This article presents a case of a 12year-old girl with embryonal orbital rhabdomyosarcoma which is a rare occurrence among adolescents and its management challenges.

\section{PRESENTATION OF CASE}

A 12-year-old girl presented to ophthalmology clinic in a hospital within Central Sarawak of East Malaysia, with a history of three months proptosis of the left eye. The proptosis was slowly progressing and painless. She has no other associated ophthalmology symptoms. Otherwise, she did not have any fever and other systemic symptoms. Her past ocular and medical history was not significant. On ocular examination, her best corrected visual acuity was 6 / 9 in the right eye and $6 / 12$ in the left eye with no relative afferent pupillary defect (RAPD). Intraocular pressure (IOP) on applanation tonometry was $12 \mathrm{mmHg}$ bilaterally. There was minimal proptosis and extraocular movement of the affected eye was minimally restricted in all directions. Otherwise, there was no eyelid swelling or erythema. Fundus examination over the affected eye was normal. Moreover, ocular examination over the fellow eye was unremarkable. An urgent computed tomography (CT) of the orbit was done and depicted the presence of hypodense mass occupying left intraconal and extraconal space. There was no bleeding or calcification of mass noted. Furthermore, left lacrimal gland was not visualised. Besides, routine blood investigations done came back as normal. She was then referred to an oculoplastic referral centre in West Malaysia for further investigations of the orbital mass. She was planned for biopsy approximately two months later after her primary school final examination.

However, approximately one month after her initial visit, her left eye proptosis progressed rapidly within the duration of one week in addition to complaint of pain and visual impairment. Clinical examination depicted no perception to light over her left eye with a positive RAPD. There was eyelid swelling, severe proptosis with diffuse orbital mass, conjunctiva chemosis and a frozen left eye. Fundus examination of the left eye depicted optic disc swelling. CT orbit was repeated and illustrated a hypodense soft tissue measuring $3.6 \mathrm{~cm} \times 2 \mathrm{~cm} \times 2.7 \mathrm{~cm}$ occupying left extra conal and intraconal spaces, indenting onto posterior aspect of the globe suggestive of left orbital rhabdomyosarcoma. No bleeding from the mass and no adjacent lymph nodes involvement. All extraocular muscles were involved except for medial rectus and inferior rectus. In addition, there was medial displacement of the optic nerve. Routine blood investigations also came back as normal.
Corresponding Author: Dr. Jacqueline Ting Yih Ling, Department of Ophthalmology, Universiti Kebangsaan Malaysia Medical Centre (UKMMC), Jalan Yaacob Latiff Cheras,

Kuala Lumpur, Malaysia.

E-mail: jac6198@hotmail.com

DOI: $10.14260 /$ jemds/2021/357

How to Cite This Article:

Ting JYL, Talib N, Mustapha M. Aggressive embryonal orbital rhabdomyosarcoma in an adolescent. J Evolution Med Dent Sci 2021;10(22):1732-1734, 10.14260/jemds/2021/357 DOI:

Submission 03-01-2021,

Peer Review 06-04-2021,

Acceptance 13-04-2021,

Published 31-05-2021.

Copyright (C) 2021 Jacqueline Yih Ling Ting et al. This is an open access article distributed under Creative Commons Attribution License [Attribution 4.0 International (CC BY 4.0)] 


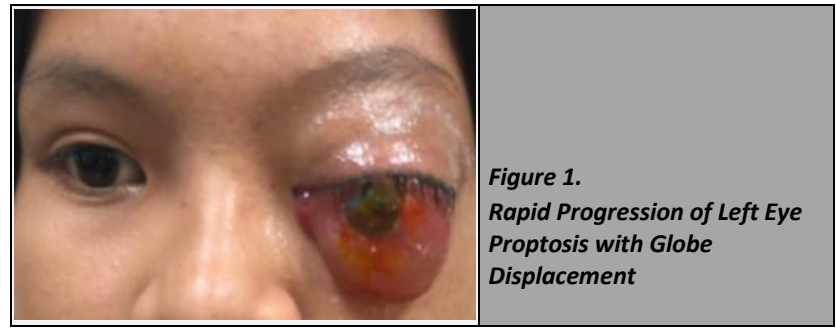

Immediate arrangement was made for her to fly from her home to the oculoplastic centre in West Malaysia which is 2 hours by flight. She then underwent left orbital mass excision via anterior orbitotomy. Intraoperative findings were left firm capsulated extraconal and intraconal mass extending to orbital apex and presence of feeder vessels. Moreover, there was no muscle infiltration. In view of difficulty confirming the histological diagnosis, the histology slide was referred to various pathologists both within the country and abroad before the final diagnosis of embryonal rhabdomyosarcoma was confirmed. Post operatively, she was planned for referral to oncologist for chemotherapy. The nearest hospital with oncology service was about 1 hour by flight from her home. Therefore, this led to a delay in initiation of chemotherapy for approximately 1 month and despite initiation of chemotherapy after that, the mass reoccurred aggressively causing massive proptosis over the same eye. The mass never regresses, and patient subsequently underwent left eye exenteration. She also continued with adjunct therapy post operation.



\section{DIFFERENTIAL DIAGNOSIS}

Lacrimal gland tumour

Idiopathic inflammatory pseudotumour

Neuroblastoma

Orbital cellulitis

\section{PATHOLOGICAL DISCUSSION}

The exact pathophysiology and origin of rhabdomyosarcoma has been a favourable topic for discussion in various scientific meetings for the past decade. ${ }^{1,2}$ It was postulated to have arisen directly from striated muscle. This may be partially true and only applicable to the pleomorphic variant. Reported cases of ocular rhabdomyosarcoma at sites such as the conjunctiva and uveal tract has led to the belief that it originates from primitive mesenchymal cells that has the ability to differentiate into striated muscles. ${ }^{3}$ Currently, there are no reported cases to suggest associations with environmental factors. There are no specific genes to suggest direct hereditary transmission of rhabdomyosarcoma.
However, it has been reported multiple associations with other genetic mutations including retinoblastoma, $R B 1$ gene on chromosome 13q14), Li-Fraumeni syndrome ( $p 53$ gene on chromosome $17 \mathrm{p} 13.1$ ), neurofibromatosis type I ( $N F 1$ gene on chromosome 17q11.2) and Costello syndrome (HRAS gene on chromosome 11p15.5). 4

Following biopsy, staging for orbital rhabdomyosarcoma is done according to the Intergroup Rhabdomyosarcoma Study (IRGS) staging system.4,5 Rhabdomyosarcoma can be further divided into four main histopathologic types, namely; embryonal, alveolar, pleomorphic and botryoid variants.4,6 It is important to identify the tissue diagnosis as the histologic subtype has been reported to affect prognosis and therefore determine the long term management of patients. ${ }^{6}$ Embryonal subtype is the most common subtype seen in orbit and usually depicts a good prognosis compared to other subtypes. ${ }^{3}$ It is composed of alternating cellular and myxoid areas. The elongated tumour cells have centrally located hyperchromatic nucleus surrounded by a considerable amount of eosinophilic cytoplasm. The rhabdomyoblastic cells may show cross striations on light microscopy representing cytoplasmic bundles of actin and myosin filaments. ${ }^{3,7}$

\section{DISCUSSION OF MANAGEMENT}

Rhabdomyosarcoma is a highly malignant neoplasm originating mainly from undifferentiated mesenchymal tissue. It is one of the commonest soft tissue neoplasms in the head and neck region, with an incidence of 4.3 cases per million. ${ }^{4}$ Approximately $10 \%$ of the cases reported occur in the orbit. It is considered a disease of young children with strong male prevalence. This article presents a case of a 12-year-old girl with embryonal orbital rhabdomyosarcoma which is a rare occurrence among adolescent and its management challenges. This case of orbital rhabdomyosarcoma stands out because of few factors; uncommon, rare among adolescent and rapid recurrence. Most of the cases reported described the characteristic presentation for orbital rhabdomyosarcoma as the rapid onset of unilateral proptosis. Shields et al. ${ }^{8}$ concluded that proptosis can present insidiously as a gradual painless process or develop rapidly within a few weeks from initial presentation. Other presentations range from eyelid swelling to signs of inflammation mimicking orbital cellulitis. ${ }^{9}$ Furthermore, visual impairment is usually minimal until the tumour becomes advanced. ${ }^{8}$ Likewise, our patient initially presented with painless proptosis which worsened rapidly over one week. In addition to visual impairment, she also presented with pain which was uncommon as they are commonly painless in nature.

Diagnosis involves a detailed history, ocular examination, imaging studies and biopsy to plan for subsequent management; a combination of surgery, irradiation, and chemotherapy. ${ }^{9}$ Although CT orbit was suggestive of lacrimal gland tumour, our patient presented with a history of four months slowly progressing proptosis and clinical examination did not depict any palpable mass to support the differential diagnosis of orbital mass which was aggressive in origin. Additionally, per parents' request, given the consideration that she was about to sit for her final year examination, biopsy was planned for two months later. Nevertheless, immediate 
arrangement for operation was made once she presented back to us with rapid progression of her condition.

Treatment and management of orbital rhabdomyosarcoma include a combination of surgery, radiotherapy and chemotherapy. ${ }^{10}$ There have been ongoing debates on the recommended surgical approach and the outcomes. In the past decades, complete excision used to be the primary treatment modality. ${ }^{11,12}$ The issue of whether tumour debulking is preferable than incisional biopsy has not been clarified. Some believe that incisional biopsy is adequate as the illness has a good prognosis following adjunct therapy regardless of amount of tissue excised. ${ }^{5}$ Recommendation by the IRSG has been in favour of diagnostic biopsy. ${ }^{13}$ In contrast, Giovanni et al. demonstrated that patients who underwent tumour debulking did slightly better in terms of overall survival rate and progression free survival rate compared to those who underwent biopsy, ${ }^{14}$ despite the differences are not statistically significant. In this case, tumour excision was done in view of the large size of the mass and to save the globe from exposure.

With the introduction of surgery combined with adjuvant chemotherapy as well as radiotherapy following the IRGS staging recommendations, it has greatly improved survival rates of patients to an average 3 years survival rate of $90 \%{ }^{5}$ However, the outcome is still greatly influenced by external factors such as delay in initiation of treatment. In this case, our patient also faced other inevitable challenges; time needed to confirm histopathological examination (HPE) diagnosis and logistic issue. Adjunct therapy is essential to prevent recurrence. ${ }^{8}$ Despite the fact that embryonal variant has a better prognosis than the other variants, her left eye exenteration was inevitable due to rapid recurrence ${ }^{14}$ In addition, follow up of patients post treatment is important in order to look for late side effects and complications of radiotherapy, chemotherapy, recurrence and secondary tumours.

In summary, this case illustrates the unpredictability progression of an orbital mass and the management challenges. Hence, orbital rhabdomyosarcoma should always be considered among adolescents with rapid-progressing orbital mass. Delay in diagnosis and inappropriate treatment can adversely affect the patient's outcome and lead to early recurrence.

\section{FINAL DIAGNOSIS}

Left orbital embryonal rhabdomyosarcoma

\section{CONCLUSIONS}

This case illustrates the unpredictability of progression of an orbital mass and the management challenges. Hence, orbital rhabdomyosarcoma should always be considered among adolescents with rapidly-progressing orbital mass. Delay in diagnosis and inappropriate treatment can adversely affect the patient's outcome and lead to early recurrence.

Financial or other competing interests: None.

Disclosure forms provided by the authors are available with the full text of this article at jemds.com.

\section{REFERENCES}

[1] Hettmer S, Wagers AJ. Muscling in: uncovering the origins of rhabdomyosarcoma. Nat Med 2010;16(2):171-3.

[2] Abraham J, Nuñez-Álvarez Y, Hettmer S, et al. Lineage of origin in rhabdomyosarcoma informs pharmacological response. Genes Dev 2014;28(14):1578-91.

[3] Karcioglu ZA, Hadjistilianou D, Rozans M, et al. Orbital rhabdomyosarcoma. Cancer Control 2004;11(5):328-33.

[4] Shields JA, Shields CL. Rhabdomyosarcoma: review for the ophthalmologist. Surv Ophthalmol 2003;48(1):39-57.

[5] Raney RB, Walterhouse DO, Meza JL, et al. Results of the intergroup rhabdomyosarcoma study group d9602 protocol, using vincristine and dactinomycin with or without cyclophosphamide and radiation therapy, for newly diagnosed patients with low-risk embryonal rhabdomyosarcoma: a report from the soft tissue sarcoma committee of the children's oncology group. J Clin Oncol 2011;29(10):1312-8.

[6] Hicks J, Flaitz C. Rhabdomyosarcoma of the head and neck in children. Oral Oncol 2002;38(5):450-9.

[7] Jurdy L, Merks JHM, Pieters BR, et al. Orbital rhabdomyosarcomas: a review. Saudi J Ophthalmol 2013;27(3):167-75.

[8] Shields JA, Shields CL. Rhabdomyosarcoma: review for the ophthalmologist. Surv Opthalmol 2003;48(1):39-57.

[9] Rao AA, Naheedy JH, Chen JYY, et al. A clinical update and radiologic review of pediatric orbital and ocular tumors. J Oncol 2013;2013:975908.

[10] Raney RB, Maurer HM, Anderson JR, et al. The Intergroup Rhabdomyosarcoma Study Group (IRSG): major lessons from the IRS-I through IRS-IV studies as background for the current IRS-V treatment protocols. Sarcoma 1900;5(1):925281.

[11] Conneely MF, Mafee MF. Orbital Rhabdomyosarcoma and simulating lesions. Neuroimaging Clin North Am 2005;15(1):121-36.

[12] Breneman JC, Lyden E, Pappo AS, et al. Prognostic factors and clinical outcomes in children and adolescents with metastatic rhabdomyosarcoma--a report from the intergroup rhabdomyosarcoma study IV. J Clin Oncol 2003;21(1):78-84.

[13] Wharam M, Beltangady M, Hays D, et al. Localized orbital rhabdomyosarcoma. An interim report of the intergroup rhabdomyosarcoma study committee. Opthalmolgy 1987;94(3):251-4.

[14] Cecchetto G, Bisogno G, Corti FD, et al. Biopsy or debulking surgery as initial surgery for locally advanced rhabdomyosarcomas in children? Cancer 2007;110(11):2561-7. 